\title{
Combined fit to the electroweak data and constraints on the Standard Model
}

\author{
Salvatore Mele* $\dagger$ \\ CERN, EP Division, CH-1211, Genève 23, Switzerland ${ }^{\ddagger}$ \\ E-mail: 'Salvatore. Meleecern.chi
}

Abstract: Precision electroweak measurements are compared to Standard Model predictions. The masses of the top quark and of the Higgs and W bosons are estimated.

More than a decade of frontier research on the nature and the manifestations of the electroweak interactions was carried on at LEP, SLC and the Tevatron. Results from a large spectrum of investigations, many of which are now close to their final stage, were discussed at this conference. The LEP collaborations reported on properties of the Z boson [i] $\left[\begin{array}{l}1 \\ 1\end{array}\right]$ and the tau lepton [2] and on forward-backward asymmetries in heavy flavour production [i3i]. Corresponding findings from SLD [0

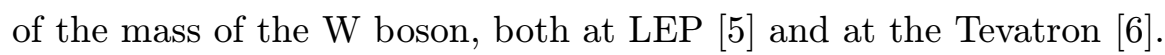

This plethora of precision measurements allows to constrain the Standard Model of the electroweak interactions through the mechanism of radiative corrections [i]. This phenomenon consists of the appearance of boson and fermion loops in the boson propagators, a classical example being the running of the electromagnetic coupling constant $\alpha$ due to fermion loops in the photon propagator. The corrections to the electroweak observables depend quadratically on the mass $m_{\mathrm{t}}$ of the top quark and logarithmically on the mass $m_{\mathrm{H}}$ of the Higgs boson.

Figure 1 presents one of the evidences for radiative corrections beyond the running of $\alpha$. The measured values of the effective electroweak mixing angle, $\sin \theta_{\text {eff }}^{2 \text { lept }}$ and the leptonic partial width of the $\mathrm{Z}$ boson, $\Gamma_{l}$, cannot be described by the running of $\alpha$ alone. Moreover they are sensitive to $m_{\mathrm{t}}$ and $m_{\mathrm{H}}$.

The synergy of the efforts of the experimental and theoretical communities has led to the implementation of a highly predictive fit procedure to compare the electroweak observables to their corresponding predictions, allowing to infer the values of the free parameters of the Standard Model. The fit is based on the ZFITTER 6.36 [i⿱⿱亠䒑女口$]$ ] and TOPAZ0 $4.4\left[\begin{array}{l}{[9} \\ {[}\end{array}\right]$ calculations. They proceed from five inputs: the values of the electromagnetic and strong coupling constants at the $\mathrm{Z}$ pole, $m_{\mathrm{t}}, m_{\mathrm{H}}$ and the mass $m_{\mathrm{Z}}$ of the $\mathrm{Z}$ boson. An additional parameter is the Fermi constant $G_{F}$, whose uncertainty is here negligible. The value of the electromagnetic coupling constant is estimated [i] $\overline{0} \overline{1}]$ from a dispersion integral

${ }^{*}$ Speaker.

${ }^{\dagger}$ on behalf of the LEP/SLD collaborations and the LEP Electroweak Working Group

${ }^{\ddagger}$ On leave of absence from INFN, Sezione di Napoli, I-80125, Napoli, Italy 
description of the cross section of $\mathrm{e}^{+} \mathrm{e}^{-}$annihilation into hadrons at low centre-of-mass energies.

For each choice of the input parameters, Standard Model predictions are obtained for the LEP and SLD electroweak observables ${ }^{1}$, the mass of the $\mathrm{W}$ boson $m_{\mathrm{W}}$, the value of the electroweak mixing angle $\sin ^{2} \theta_{W}$ as measured in neutrino-nucleon scattering and, for the first time, the atomic parity violation as measured for cesium atoms. Those values are compared by means of a $\chi^{2}$ fit to the corresponding exper-

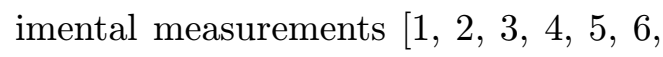

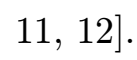

Six of the electroweak observables under investigation deserve a special mention: $A_{\mathrm{FB}}^{0, \ell}$, the tau polarisation, the leftright asymmetry measured at SLD, $A_{\mathrm{FB}}^{0, \mathrm{c}}$, $A_{\mathrm{FB}}^{0, \mathrm{~b}}$ and $<Q_{\mathrm{FB}}>$. Figure 2 presents the values of $\sin \theta_{\text {eff }}^{2 \text { lept }}$ that they yield [i]', 2 three measurements, based on leptons, agree with each other with a $\chi^{2} /$ d.o.f of $1.6 / 2$, corresponding to a $44.3 \%$ Confidence Level (CL), as do the last three

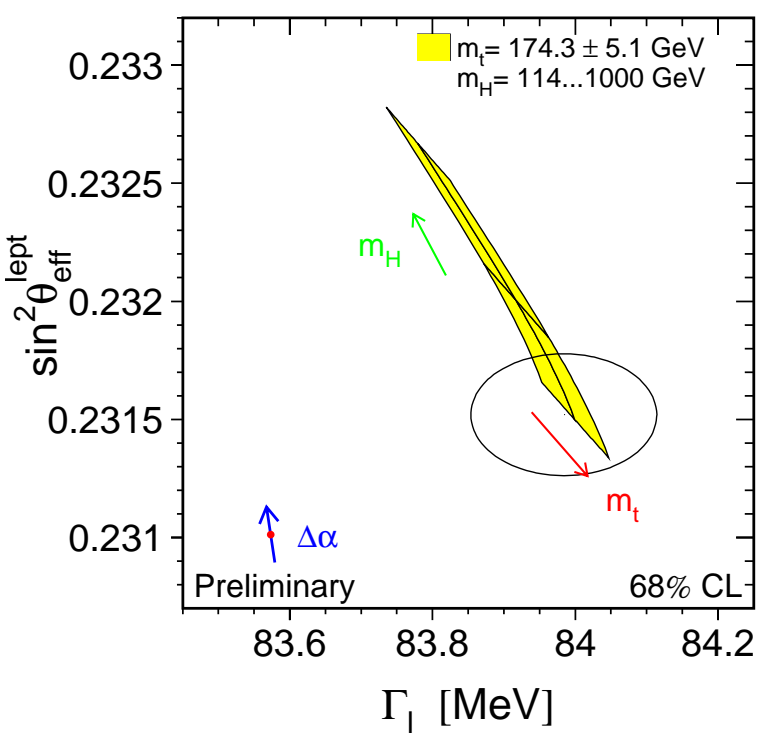

Figure 1: Measurement at $68 \%$ of Confidence level in the $\left(\Gamma_{l}, \sin \theta_{\text {eff }}^{\text {lept }}\right)$ plane. The point labelled $\Delta \alpha$ represents the predictions in presence of the $\alpha$ running only, the arrow being an additional variation within its uncertainties, while the band is the prediction for the indicated ranges of $m_{\mathrm{t}}$ and $m_{\mathrm{H}}$.

with $\chi^{2} /$ d.o. $f=0.3 / 2(84.6 \% \mathrm{CL})$. The global agreement is poor, $\chi^{2} /$ d.o. $f=12.8 / 5(2.5 \%$ CL), but no exhaustive conclusion can be drawn from this observation.

The full fit has a $\chi^{2} /$ d.o.f of $22.9 / 15$ corresponding to a $8.6 \%$ CL. The two largest contribution to this $\chi^{2}$ stem from $A_{\mathrm{FB}}^{0, \mathrm{~b}}$ and $A_{\mathrm{FB}}^{0, \mathrm{c}}$, as presented in figure 3 in terms of the "pull", that quantifies the number of experimental standard deviation of which each measurement deviates from the predictions of the fit. Table 1 and figures $4 \mathrm{a}-\mathrm{c}$ compare

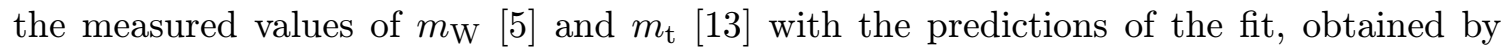
releasing the corresponding constraints. A good agreement is observed for $m_{\mathrm{t}}$ and a worse one for $m_{\mathrm{W}}$, still confirming the internal consistency of the Standard Model. Predictions for $m_{\mathrm{H}}$ are also given.

Using all constraints, the mass of the Higgs boson is predicted (figure 4d) as:

$$
m_{\mathrm{H}}=88_{-35}^{+53} \mathrm{GeV}, \quad m_{\mathrm{H}}<196 \mathrm{GeV}(95 \% \mathrm{CL}) .
$$

\footnotetext{
${ }^{1}$ Those include $m_{\mathrm{Z}}$, the total and leptonic widths of the $\mathrm{Z}$ boson, the cross section for the production of hadrons at the $\mathrm{Z}$ peak, the lepton and heavy quark asymmetries and forward-backward asymmetries at the $\mathrm{Z}$ pole, $A_{\mathrm{FB}}^{0,(\ell, \mathrm{b}, \mathrm{c})}$, together with the inclusive hadronic event forward backward asymmetry, $\left\langle Q_{\mathrm{FB}}>\right.$, and the ratio of heavy flavour production with respect to hadrons.
} 


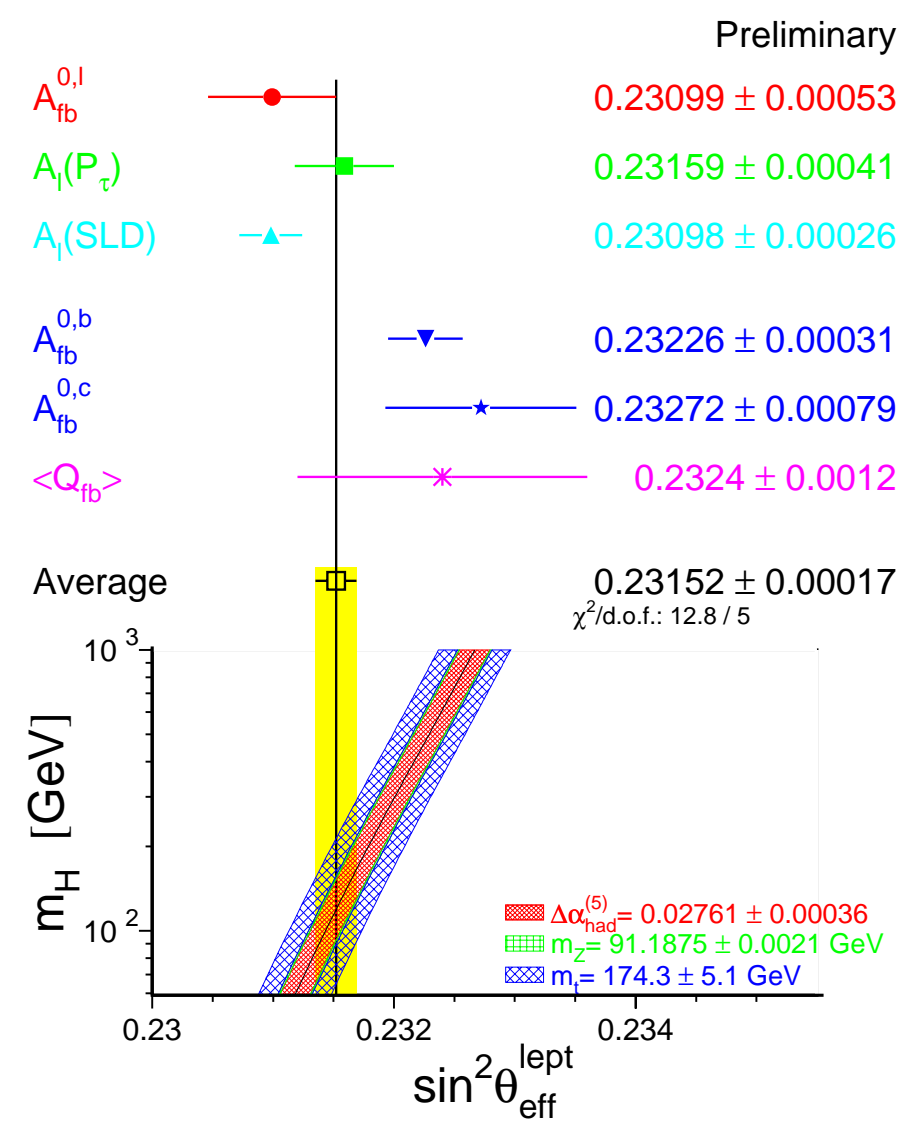

Figure 2: Values of $\sin \theta_{\text {eff }}^{\text {lept }}$ extracted from lepton and hadron observables and predicted dependence on $m_{\mathrm{H}}$.

\begin{tabular}{|c|c|c|c|c|}
\hline & $m_{\mathrm{W}}$ free & $m_{\mathrm{t}}$ free & $m_{\mathrm{W}}$ and $m_{\mathrm{t}}$ free & Data \\
\hline$m_{\mathrm{W}}(\mathrm{GeV})$ & $80.373 \pm 0.023$ & - & $80.363 \pm 0.032$ & $80.451 \pm 0.033[\overline{5}[\overline{5}]$ \\
\hline$m_{\mathrm{t}}(\mathrm{GeV})$ & - & $181_{-9}^{+11}$ & $169_{-9}^{+12}$ & $174.3 \pm 5.1\left[1133_{1}^{0}\right]$ \\
\hline$m_{\mathrm{H}}(\mathrm{GeV})$ & $108_{-44}^{+70}$ & $126_{-69}^{+182}$ & $81_{-40}^{+109}$ & $<114.1(95 \%$ CL $)[\overline{1} \overline{1} \overline{6}]$ \\
\hline$\chi^{2} /$ d.o.f & $19.1 / 13$ & $22.6 / 14$ & $18.9 / 12$ & 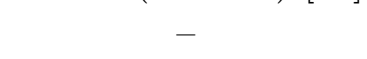 \\
\hline
\end{tabular}

Table 1: Results of the fits without the $m_{\mathrm{W}}$ and $m_{\mathrm{t}}$ constraints compared with the measurements.

All estimates take into account the theory uncertainties from higher order and QCD cor-

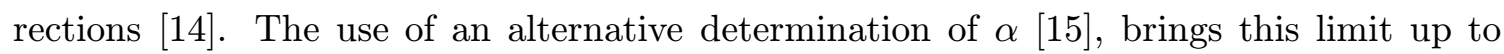

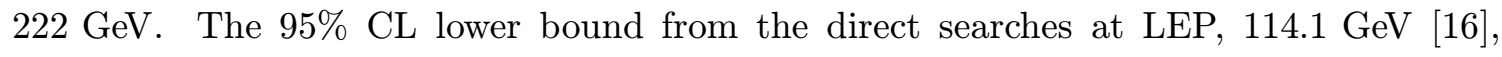
further corners the allowed range for $m_{\mathrm{H}}$.

The predictive power of the fit will be enhanced by the reduction of the uncertainty on $m_{\mathrm{W}}$ at LEP [is and by new measurements of $m_{\mathrm{W}}$ and $m_{\mathrm{t}}$ at the upgraded Tevatron [i] as well as by inputs from neutrino-nucleon scattering. In addition, comparing these experimental results with the predictions will be of paramount importance: the goodness of the fit is at present acceptable though not excellent and further deviations might herald New Physics beyond the Standard Model. 


\begin{tabular}{|c|c|c|c|}
\hline & \multicolumn{2}{|c|}{ Summer 2001} & \multirow[b]{2}{*}{ 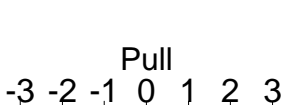 } \\
\hline & Measurement & Pull & \\
\hline$\Delta \alpha_{\text {had }}^{(5)}\left(m_{z}\right)$ & $0.02761 \pm 0.00036$ & -.35 & - \\
\hline$m_{z}[\mathrm{GeV}]$ & $91.1875 \pm 0.0021$ & .03 & \\
\hline$\Gamma_{\mathrm{Z}}[\mathrm{GeV}]$ & $2.4952 \pm 0.0023$ & -.48 & - \\
\hline$\sigma_{\text {had }}^{0}[\mathrm{nb}]$ & $41.540 \pm 0.037$ & 1.60 & $\boldsymbol{\infty}$ \\
\hline $\mathrm{R}_{\mathrm{l}}$ & $20.767 \pm 0.025$ & 1.11 & 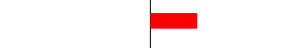 \\
\hline$A_{\mathrm{fb}}^{0, I}$ & $0.01714 \pm 0.00095$ & .69 & 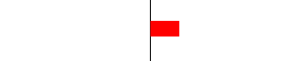 \\
\hline$A_{1}\left(P_{\tau}\right)$ & $0.1465 \pm 0.0033$ & -.54 & - \\
\hline $\mathrm{R}_{\mathrm{b}}$ & $0.21646 \pm 0.00065$ & 1.12 & \\
\hline $\mathrm{R}_{\mathrm{c}}$ & $0.1719 \pm 0.0031$ & -.12 & 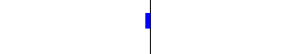 \\
\hline$A_{f b}^{0, b}$ & $0.0990 \pm 0.0017$ & -2.90 & \\
\hline$A_{\mathrm{fb}}^{0, \mathrm{c}}$ & $0.0685 \pm 0.0034$ & -1.71 & \\
\hline$A_{b}$ & $0.922 \pm 0.020$ & -.64 & - \\
\hline$A_{c}$ & $0.670 \pm 0.026$ & .06 & \\
\hline$A_{1}(S L D)$ & $0.1513 \pm 0.0021$ & 1.47 & \\
\hline $\sin ^{2} \theta_{\text {eff }}^{\text {lept }}\left(Q_{\mathrm{fb}}\right)$ & $0.2324 \pm 0.0012$ & .86 & 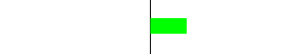 \\
\hline $\mathrm{m}_{\mathrm{W}}^{(\mathrm{LEP})}[\mathrm{GeV}]$ & $80.450 \pm 0.039$ & 1.32 & 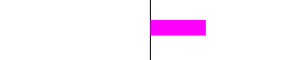 \\
\hline$m_{t}[\mathrm{GeV}]$ & $174.3 \pm 5.1$ & -.30 & - \\
\hline $\mathrm{m}_{\mathrm{W}}^{(\mathrm{TEV})}[\mathrm{GeV}]$ & $80.454 \pm 0.060$ & .93 & $=$ \\
\hline $\sin ^{2} \theta_{w}(v N)$ & $0.2255 \pm 0.0021$ & 1.22 & 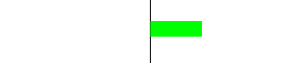 \\
\hline$Q_{W}(C s)$ & $-72.50 \pm 0.70$ & .56 & L \\
\hline
\end{tabular}

Figure 3: Input values for the fit and their deviations from the predictions

\section{Acknowledgments}

It has been a privilege to share those years of physics at LEP with all my colleagues, too many to be listed here. I am grateful to Martin Grünewald for his help in the preparation of my talk.

\section{References}

[1] M. Paganoni in Proceedings of the EPS International Conference on High Energy Physics, Budapest, 2001 (D. Horvath, P. Levai, A. Patkos, eds.), JHEP (http://jhep.sissa.it/) Proceedings Section, PrHEP-hep2001/111.

[2] P. Casado, PrHEP-hep2001/112.

[3] P. Hansen, PrHEP-hep2001/113.

[4] V. Serbo, PrHEP-hep2001/114.

[5] H. Ruiz, PrHEP-hep2001/121.

[6] C. Gerber, PrHEP-hep2001/126.

[7] G. Altarelli et al., CERN Report 89-08 (1989) and references therein. 

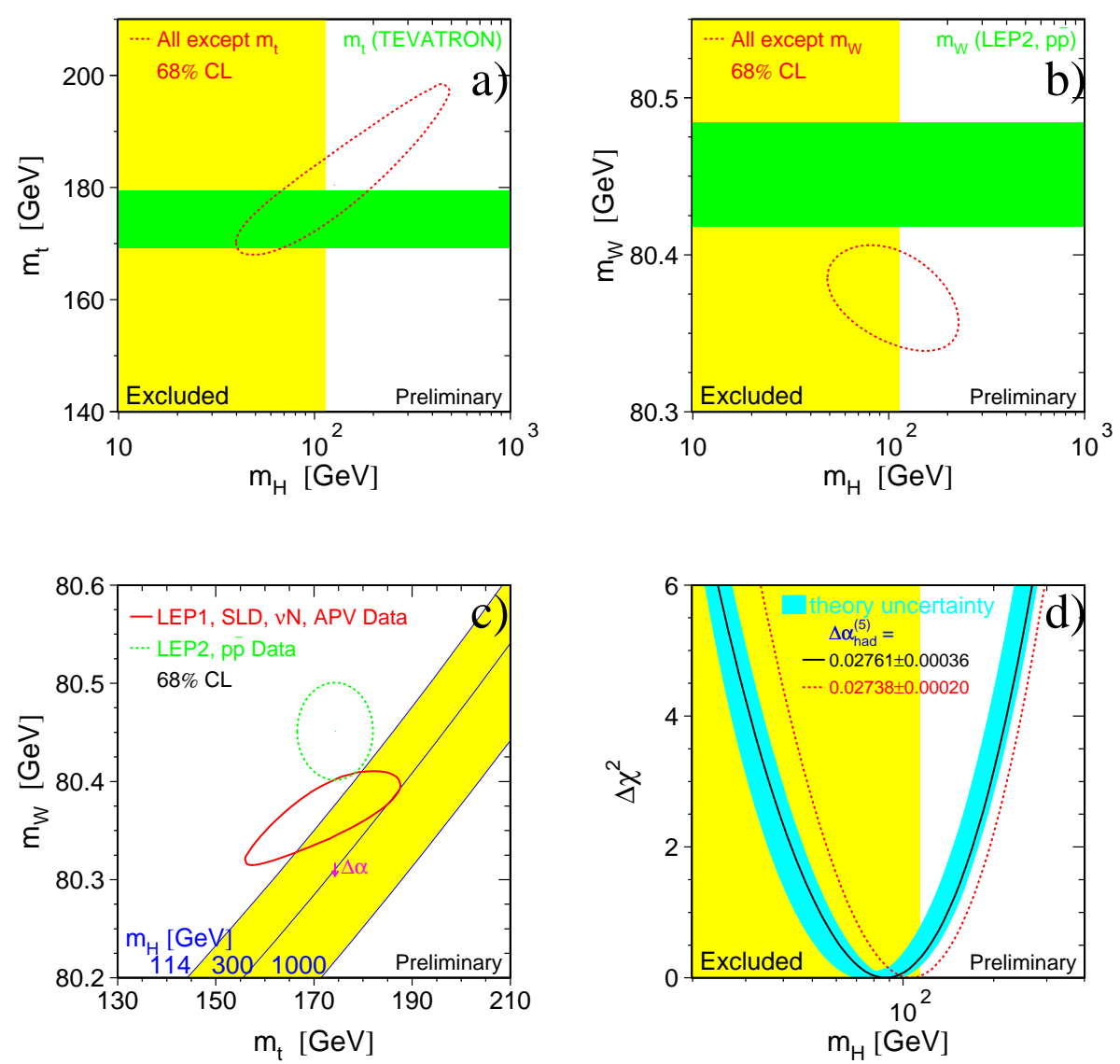

Figure 4: Fit predictions compared to the measurements for a) $m_{\mathrm{W}} v s . m_{\mathrm{H}}$, b) $m_{\mathrm{t}} v s . m_{\mathrm{H}}$ and

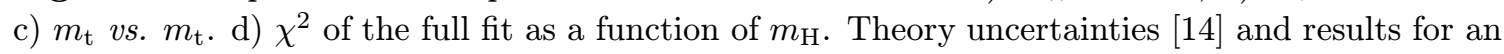
alternative value of $\alpha$ [1는

[8] D.Y. Bardin et al., 'Comput. Physs. Commun. 133 $(200 \overline{1}) 22 \overline{2} \overline{-}$.

[9] G. Montagna et al., Comput. Phys. Commun. 117 (1999) 278.

[10] H. Burkhardt and B. Pietrzyk, 'Phys. Lett. B

[11] K. McFarland et al., CCFR/NuTeV Coll. Eur. Phys.

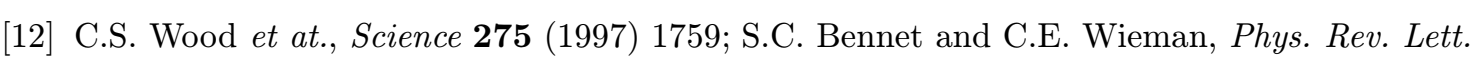
- - 82 (1999) 2484

[13] C. Caso et al., Eur. Phys. J. 15 (2000) 1.1.

[14] D. Bardin et al., hep-ph/9902452; D. Bardin et al., CERN Report 95-03 (1995); A. Freitas et

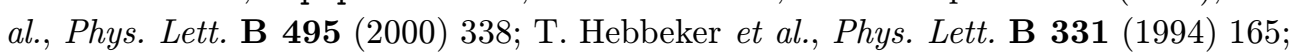

P.A. Raczka and A. Szymacha, Phys. Rev D 54 (1996) 3073; D.E Soper and

L.R. Surguladze, 'Physs. Rev. D $54(1996)=4566^{\prime}$

[15] A.D. Martin et al., 'Phys. Lett. B $\mathbf{4} \mathbf{9 2}(2000) 69_{r}^{\prime}$

[16] J. Marco, PrHEP-hep2001/128.

[17] G. Chiarelli, PrHEP-hep2001/131. 